\title{
MODULI OF FLAT SU(3)-BUNDLES OVER A KLEIN BOTTLE
}

\author{
ThOMAS JOHn BAIRD
}

\begin{abstract}
In this short note, we compute the Betti numbers of the moduli stack of flat $S U(3)$-bundles over a Klein bottle. We also handle the general compact group case over $\mathbb{R} P^{2}$. In all cases the cohomology is found to be equivariantly formal, supporting a conjecture from the author's doctoral thesis. Our results also verify conjectural formulas obtained by Ho-Liu using Yang-Mills Morse theory.
\end{abstract}

\section{Introduction}

Let $M$ be a smooth, compact nonorientable 2-manifold. For a choice of Lie group $G$, one may study the set of group homomorphisms $\operatorname{Hom}\left(\pi_{1}(M), G\right)$, topologized using the compact open topology. The group $G$ acts continuously on $\operatorname{Hom}\left(\pi_{1}(M), G\right)$ by conjugation, and we deem two homomorphisms equivalent if they lie in the same orbit. The quotient stack $\left[\operatorname{Hom}\left(\pi_{1}(M), G\right) / G\right]$ is well known to be isomorphic to the topological moduli stack of flat $G$-bundles over $M$ (see [4] for example).

In previous work [1], the author computed the stack cohomology

$$
H_{\text {stack }}^{*}\left(\left[\operatorname{Hom}\left(\pi_{1}(M), S U(2)\right) / S U(2)\right] ; \mathbb{Q}\right)=H_{S U(2)}^{*}\left(\operatorname{Hom}\left(\pi_{1}(M), S U(2)\right) ; \mathbb{Q}\right)
$$

in the rank 2 case, where the conjugation action was found to be equivariantly formal (Recall that a $G$-space $X$ is called equivariantly formal if $H_{G}^{*}(X) \cong H^{*}(X) \otimes H^{*}(B G)$ as graded vector spaces). This motivated the conjecture that equivariant formality remains true when $S U(2)$ is replaced by any compact connected Lie group $G$.

In this paper we compute rational Betti numbers and establish the conjecture in the case that $G=S U(3)$ and $M$ is the Klein bottle. We also establish the conjecture for $M=\mathbb{R} P^{2}$ and arbitrary compact, connected $G$.

The case of $G=S U(3)$ has already been studied by Ho-Liu [6], using YangMills Morse theory. The Yang-Mills functional determines a Morse stratification of the space $\mathcal{A}$ of connections, whose semistable stratum deformation retracts onto the space $\mathcal{A}_{\text {flat }}$ of flat connections. Ho and Liu prove that the normal bundles of the Morse strata have vanishing equivariant Euler classes and introduce the term locally antiperfect to describe this property. They conjecture that the stratification is also globally antiperfect and produce under this assumption candidate Poincaré series for the moduli stacks. For a $G$-space $X$, the Poincaré series $P_{t}^{G}(X)$ is defined by

$$
P_{t}^{G}(X)=\sum_{i=0}^{\infty} \operatorname{dim}\left(H_{G}^{i}(X ; \mathbb{Q})\right) t^{i}
$$

Let $\Sigma_{n}$ denoted the connected sum of $n+1$ copies of $\mathbb{R} P^{2}$. Ho-Liu propose that

Received by the editors January 10, 2009. 


$$
P_{t}^{S U(3)}\left(\operatorname{Hom}\left(\pi_{1}\left(\Sigma_{n}\right), S U(3)\right)\right) \stackrel{!}{=} \frac{P_{t}(S U(3))^{n}+\left(1+t^{2}+t^{4}\right)\left(t^{3}+2 t^{4}+t^{5}\right)^{n}}{\left(1-t^{4}\right)\left(1-t^{6}\right)}
$$

In this paper we confirm (1) in the cases $n=0,1$.

Notational convention: Unless otherwise stated, cohomology is singular with $\mathbb{Q}$ coefficients.

\section{Equivariant formality}

We review the notion of equivariant formality.

Let $G$ be a compact, connected Lie group with universal $G$-bundle $G \rightarrow E G \rightarrow B G$ and let $X$ denote a compact, Hausdorff $G$-space. The equivariant cohomology of a $G$-space $X$ is defined to be the cohomology of the associated fibre bundle $X \rightarrow$ $E G \times_{G} X \rightarrow B G$,

$$
H_{G}^{*}(X ; \mathbb{Q})=H^{*}\left(E G \times_{G} X ; \mathbb{Q}\right) .
$$

The Serre spectral sequence $\left(E_{*}^{p, q}\right)$ of the fibration $E G \times{ }_{G} X$ satisfies $E_{2}^{p, q} \cong H^{p}(X) \otimes$ $H^{q}(B G)$ and converges to $H_{G}^{*}(X)$. If the spectral sequence collapses at page 2 , then $H_{G}^{*}(X) \cong H^{*}(X) \otimes H^{*}(B G)$ as graded vector spaces and we say the $G$-space $X$ is equivariantly formal. The cohomology ring $H^{*}(B G)$ is a polynomial ring with even degree generators, so $H^{\text {odd }}(B G)=0$. Consequently, if $H^{\text {odd }}(X)=0$ then $X$ must be equivariantly formal.

Let $T \subset G$ denote a maximal torus in $G$. The $G$-space $X$ is $G$-equivariantly formal if and only if it is $T$-equivariantly formal under the restricted $T$-action. If $X^{T}$ denotes the $T$-fixed point set, by [3] IV 5.5 we have

$$
\operatorname{dim} H^{*}(X) \geq \operatorname{dim} H^{*}\left(X^{T}\right)
$$

with equality if and only if $X$ is equivariantly formal.

\section{The projective plane}

The projective plane has fundamental group $\pi_{1}\left(\mathbb{R} P^{2}\right) \cong \mathbb{Z} / 2 \mathbb{Z}$. It follows that $\operatorname{Hom}\left(\pi_{1}\left(\mathbb{R} P^{2}\right), G\right) \cong\left\{g \in G \mid g^{2}=\mathbb{1}\right\}$ is exactly the set of square roots of the identity element.

Proposition 3.1. Let $G$ denote a compact, connected Lie group. The G-space $\operatorname{Hom}\left(\pi_{1}\left(\mathbb{R} P^{2}\right), G\right)$ is equivariantly formal.

Proof. The set of square roots of $\mathbb{1} \in G$ is clearly invariant under conjugation. Every conjugacy class intersects a maximal torus $T \subset G$ and the number of roots of $\mathbb{1}$ in $T$ is finite, equal to $2^{\operatorname{rank}(T)}$. So $\operatorname{Hom}\left(\pi_{1}\left(\mathbb{R} P^{2}\right), G\right)$ is a finite union of homogeneous spaces of the form $G / H$ where $\operatorname{rank}(H)=\operatorname{rank}(G)$. Such $G$-spaces are equivariantly formal because $H^{o d d}(G / H)=0$ (see [5] pg. 467).

Example 1. The space $\operatorname{Hom}\left(\pi_{1}\left(\mathbb{R} P^{2}\right), U(n)\right)$ is isomorphic to $\left\{A \in U(n) \mid A^{2}=\mathbb{1}\right\}$. Since $A^{2}=\mathbb{1}$ implies that the eigenvalues of $A$ are \pm 1 , by choosing (say) the +1 eigenspace, we obtain an isomorphism to a union of Grassmanians:

$$
\operatorname{Hom}\left(\pi_{1}\left(\mathbb{R} P^{2}\right), U(n)\right) \cong \coprod_{k=0}^{n} G r_{k}\left(\mathbb{C}^{n}\right)
$$


similarly

$$
\operatorname{Hom}\left(\pi_{1}\left(\mathbb{R} P^{2}\right), S U(n)\right) \cong \coprod_{\mathrm{k} \text { even }} G r_{k}\left(\mathbb{C}^{n}\right)
$$

In particular,

$$
P_{t}\left(\operatorname{Hom}\left(\pi_{1}\left(\mathbb{R} P^{2}\right), S U(3)\right)\right)=1+\left(1+t^{2}+t^{4}\right) .
$$

confirming (1) when $n=0$.

\section{The Klein bottle}

In this section we consider the moduli stack of rank 3 bundles over a Klein bottle, $K l$. It will be useful to pass between structure groups $U(3)$ and $S U(3)$ using the following Lemma:

Proposition 4.1. We have an equation of Poincaré polynomials

$$
P_{t}\left(H o m_{i d}\left(\pi_{1}(K l), U(3)\right)\right)=(1+t) P_{t}\left(H o m\left(\pi_{1}(K l), S U(3)\right)\right),
$$

where $\mathrm{Hom}_{i d}\left(\pi_{1}(K l), U(3)\right)$ denotes the connected component of $\mathrm{Hom}\left(\pi_{1}(K l), U(3)\right)$ containing the trivial homomorphism.

We postpone the proof of 4.1 until the end of the section.

The fundamental group of the Klein bottle $K l$ has a presentation

$$
\pi_{1}(K l) \cong\left\{a_{0}, a_{1} \mid a_{0}^{2}=a_{1}^{2}\right\} .
$$

It follows that

$$
\operatorname{Hom}\left(\pi_{1}(K l), G\right) \cong\left\{\left(g_{0}, g_{1}\right) \in G^{2} \mid g_{0}^{2}=g_{1}^{2}\right\} .
$$

For the rest of this section, set $G:=S U(3)$ and $Z:=\operatorname{Hom}\left(\pi_{1}(K l), G\right)$. The main result of this paper is

Theorem 4.2. The space Hom $\left(\pi_{1}(K l), S U(3)\right)$ has Poincaré polynomial

$$
P_{t}\left(H o m\left(\pi_{1}(K l), S U(3)\right)\right)=\left(1+t^{3}\right)\left(1+t^{5}\right)+\left(1+t^{2}+t^{4}\right)\left(t^{3}+2 t^{4}+t^{5}\right) .
$$

Corollary 4.3. The conjugation action of $S U(3)$ on $\operatorname{Hom}\left(\pi_{1}(K l), S U(3)\right)$ is equivariantly formal. Consequently

$$
P_{t}^{S U(3)}\left(H o m\left(\pi_{1}(K l), S U(3)\right)\right)=\frac{\left(1+t^{3}\right)\left(1+t^{5}\right)+\left(1+t^{2}+t^{4}\right)\left(t^{3}+2 t^{4}+t^{5}\right)}{\left(1-t^{4}\right)\left(1-t^{6}\right)}
$$

in accordance with (1).

Proof. Let $T \subset G=S U(3)$ be a maximal torus. The $T$-fixed point set is

$$
\operatorname{Hom}\left(\pi_{1}(K l), G\right)^{T}=\operatorname{Hom}\left(\pi_{1}(K l), T\right) .
$$

Any such homomorphism must pass through the abelianization

$$
A b\left(\pi_{1}(K l)\right) \cong H_{1}(K l ; \mathbb{Z}) \cong \mathbb{Z} \oplus \mathbb{Z} / 2 \mathbb{Z}
$$

, so

$$
\operatorname{Hom}\left(\pi_{1}(K l), G\right)^{T} \cong T \times T_{2},
$$

where $T_{2} \cong \mathbb{Z} / 2 \mathbb{Z} \oplus \mathbb{Z} / 2 \mathbb{Z}$ is the 2 -torsion subgroup of $T$. In particular

$$
\operatorname{dim}\left(\operatorname{Hom}\left(\pi_{1}(K l), G\right)^{T}\right)=16=\operatorname{dim}\left(\operatorname{Hom}\left(\pi_{1}(K l), G\right)\right)
$$

which by (2) proves equivariant formality. Thus $P_{t}^{S U(3)}\left(H o m\left(\pi_{1}(K l), S U(3)\right)\right)=$ $P_{t}\left(H o m\left(\pi_{1}(K l), S U(3)\right)\right) P_{t}(B S U(3))$, giving rise to $(3)$. 
We will compute the Poincaré polynomial for $Z$ using the long exact sequence of a pair. Let $A \subset Z$ denote the subset of pairs $\left(k_{0}, k_{1}\right)$ such that $k_{0}$ and $k_{1}$ lie in some common maximal torus. The subspace $A$ is preserved by the $G$-action, and the stabilizers of points in $A$ all contain maximal tori of $G$. Applying ( [2], Theorem 3.3) we produce a $G$-equivariant cohomology isomorphism

$$
\phi: G / T \times_{W} Z^{T} \rightarrow A .
$$

Here $T \subset G$ is a maximal torus, $G / T$ is a coset space, $Z^{T}$ is the set of $T$-fixed points, $W=N(T) / T$ is the Weyl group and $G / T \times_{W} Z^{T}$ is the orbit space of the product $G / T \times Z^{T}$ under the natural diagonal $W$ action. The map $\phi$ sends $\phi(g T, z)=g \cdot z$.

Lemma 4.4. Let $W=N(T) / T$ act on $T_{2}:=\left\{t \in T \mid t^{2}=\mathbb{1}\right\}, T$ and $G / T$ in the usual way. There is a cohomology isomorphism

$$
H^{*}(A ; \mathbb{Q}) \cong H^{*}\left(G / T \times_{W}\left(T \times T_{2}\right) ; \mathbb{Q}\right)
$$

with Poincaré polynomial

$$
P_{t}(A)=\left(1+t^{3}\right)\left(1+t^{5}\right)+\left(1+t^{2}+t^{4}\right)(1+t)\left(1+t^{3}\right)
$$

Proof. The $T$-fixed point set $Z^{T}$ consists of pairs $\left(g_{0}, g_{1}\right) \in T^{2}$ such that $t_{0}^{2}=t_{1}^{2}$ which is equivalent to $t_{0} t_{1} \in T_{2}$, so $Z^{T} \cong T \times T_{2}$. Equation (5) then follows from (4).

Choose the maximal torus to be the diagonal matrices in $S U(3)$. Then $T_{2}$ consists of the four matrices with diagonal entries $\{(1,1,1),(-1,1,1),(1,-1,1),(1,1,-1)\}$. The Weyl group $W \cong S_{3}$ acts on $T_{2}$ by fixing the identity and permuting the remaining three element. It follows that $G / T \times_{W}\left(T \times T_{2}\right)$ has two components. The first component $G / T \times_{W} T$ has cohomology isomorphic to $S U(3)$ and contributes the first term of (6). The second component is $G / T \times_{\mathbb{Z}_{2}} T$ where the $\mathbb{Z}_{2}$ action is generated by any of the order two elements in $W$. The Poincaré polynomials we need to know are $P_{t}(G / T)=\left(1+2 t^{2}+2 t^{4}+t^{6}\right), P_{t}\left((G / T) / \mathbb{Z}_{2}\right)=P_{t}\left(\mathbb{C} P^{2}\right)=1+t^{2}+t^{4}, P_{t}(T)=(1+t)^{2}$ and $P_{t}\left(T / \mathbb{Z}_{2}\right)=1+t$. The result follows by a simple calculation.

Equation (5) provides a description of $A$ that can be generalized to any compact Lie group $G$. The more complicated part is describing the complement $Z-A$.

Lemma 4.5. The Poincaré polynomial of the pair $(Z, A)$ satisfies

$$
P_{t}(Z, A) \leq(1+t)\left(t^{1}+t^{3}+t^{6}+t^{8}\right)
$$

where the notation $p(t) \leq q(t)$ means that $q(t)-p(t)$ has no negative coefficients.

Proof. We will show that $Z-A$ is a 9-dimensional orientable manifold, so by Poincaré duality $H^{*}(Z, A) \cong H^{(9-*)}(Z-A)$ and we can focus instead on $Z-A$.

A pair $(g, h) \in Z-A$ is characterized by $g, h \in S U(3), g h \neq h g, g^{2}=h^{2}$. Since $g$ and $h$ both commute with $g^{2}$ but not with each other, we deduce that $g^{2}$ must have an eigenvalue $\lambda^{2}$ of multiplicity greater than one, and that $g$ and $h$ have eigenvalues $\lambda,-\lambda, \lambda^{-2}$. It is easily verified that $g, h$ must share an eigenvector $\bar{x}$ such that $g \bar{x}=h \bar{x}=\lambda^{-2} \bar{x}$ and that up to scalar multiplication $\bar{x}$ is the only common eigenvector of $g$ and $h$.

Now let $P\left(\mathbb{C}^{3}\right)$ denote the set of Hermitian, rank 1 projection operators on $\mathbb{C}^{3}$. We define the flag variety $F l_{3}:=\left\{p=\left(p_{1}, p_{2}, p_{3}\right) \in P\left(\mathbb{C}^{3}\right) \mid p_{i} p_{j}=\delta_{i, j} p_{i}\right\}$ where $\delta_{i, j}$ denotes the Dirac delta. Define $\psi: F l_{3} \times U(1) \rightarrow S U(3)$ by $\psi(p, \lambda)=\lambda p_{1}-\lambda p_{2}+\lambda^{2} p_{3}$. 
Define $Y:=\left\{(p, q) \in F l_{3} \mid p_{3}=q_{3}\right.$ and $\left.p_{1} q_{1} \neq q_{1} p_{1}\right\}$. Then by the above discussion, the map $\rho: Y \times U(1) \rightarrow Z-A$ defined by $\rho((p, q), \lambda)=(\psi(p, \lambda), \psi(q, \lambda))$ is a two sheeted cover. It follows that the induced map $H^{*}(Z-A) \hookrightarrow H^{*}(Y \times U(1))$ is injective. We can therefore obtain upperbounds of the Betti numbers of $Z-A$ by computing $H^{*}(Y)$.

Let $\pi: Y \rightarrow F l_{3}$ denote the projection $\pi(p, q)=p$. An element $q$ in the fibre $\pi^{-1}(p)$ is uniquely determined by a choice $q_{1} \in P\left(\mathbb{C}^{3}\right)$ with $q_{1} p_{3}=0$ and $q_{1} \neq p_{1}, p_{2}$. Thus $\pi$ has fibres isomorphic to $\mathbb{C} P^{1}-2 p t s \cong \mathbb{C}^{*}$. In fact, if $L_{1}, L_{2}, L_{3}$ denote the tautological line bundles over $F l_{3}$, it follows that $Y$ is isomorphic to $\operatorname{Hom}\left(L_{1}, L_{2}\right)$ minus the zero section. We see that $Y \times U(1)$ is a 9-dimensional orientable manifold. The Serre spectral sequence to the $\mathbb{C}^{*}$-bundle $Y \rightarrow F l_{3}$ has $E_{2}$ page:

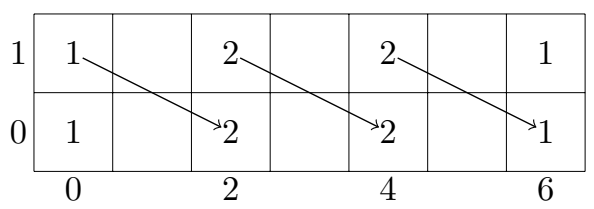

The boundary map from $E_{2}^{p, 1} \cong H^{p}\left(F l_{3}\right)$ to $E_{2}^{p+2,0} \cong H^{p+2}\left(F l_{3}\right)$ is simply cup product by the first Chern class of $\operatorname{Hom}\left(L_{1}, L_{2}\right)$. Here

$$
\begin{gathered}
H^{*}\left(F l_{3}\right) \cong Q\left[x_{1}, x_{2}, x_{3}\right] /(\text { symmetric polynomials }), \\
c_{1}\left(\operatorname{Hom}\left(L_{1}, L_{2}\right)\right)=x_{2}-x_{1}
\end{gathered}
$$

\begin{tabular}{|c|c|c|c|}
\hline 0 & 0 & 1 & 1 \\
\hline 1 & 1 & 0 & 0 \\
\hline
\end{tabular}

and the $E_{3}$ page is:

So $P_{t}(Y)=1+t^{2}+t^{5}+t^{7}$ so $P_{t}(Z-A) \leq\left(1+t^{2}+t^{5}+t^{7}\right)(1+t)$ by the Kunneth theorem, and Poincaré duality completes the proof.

In fact we will show in the course of proving Theorem 4.2 that (7) is an equality. We need one more Lemma for our calculation.

Lemma 4.6. Modulo terms of order 4 or higher,

$$
P_{t}(Z)=1+2 t^{3}\left(\bmod t^{4}\right) .
$$

Proof. Let $\mathcal{A}$ denote the affine space of $U(3)$-connections on the trivial $\mathbb{C}^{3}$ bundle over $K l, \mathcal{A}_{\text {flat }}$ the subspace of flat connections and let $\mathcal{G}_{b}$ denote the group of gauge transformations based at one point. According to ( [1] proof of Theorem 1.1) we have an injection $H_{\mathcal{G}_{b}}^{*}(\mathcal{A}) \rightarrow H_{\mathcal{G}_{b}}^{*}\left(\mathcal{A}_{\text {flat }}\right)$ and an isomorphism $H_{\mathcal{G}_{b}}^{*}(\mathcal{A}) \cong H^{*}(U(3))$, so

$$
P_{t}^{\mathcal{G}_{b}}\left(\mathcal{A}_{\text {flat }}\right)=(1+t)\left(1+t^{3}\right)\left(1+t^{5}\right)+t^{-1} P_{t}^{\mathcal{G}}\left(\mathcal{A}, \mathcal{A}_{\text {flat }}\right)
$$

It was proven in [7] and [8] that the Yang-Mills functional determines a Morse stratification, $\mathcal{A}=\bigcup_{\mu=0}^{\infty} \mathcal{A}_{\mu}$, where $\mathcal{A}_{\mu}$ is a connected submanifold with codimension $4 \mu$ and orientable normal bundle and for which the open stratum $\mathcal{A}_{0}$ contains $\mathcal{A}_{\text {flat }}$ 
as a deformation retract. We deduce that $P_{t}^{\mathcal{G}_{b}}\left(\mathcal{A}, \mathcal{A}_{\text {flat }}\right)=P_{t}^{\mathcal{G}_{b}}\left(\mathcal{A}, \mathcal{A}_{0}\right)=t^{4}\left(\bmod t^{5}\right)$, so

$$
P_{t}^{\mathcal{G}_{b}}\left(\mathcal{A}_{\text {flat }}\right)=(1+t)\left(1+t^{3}\right)\left(1+t^{5}\right)+t^{3}=1+t+2 t^{3}\left(\bmod t^{4}\right)
$$

Finally, holonomy determines a homeomorphism $\mathcal{A}_{\text {flat }} / \mathcal{G}_{0} \cong H_{o m}{ }_{i d}\left(\pi_{1}(K l), U(3)\right)$, so the lemma follows by applying Lemma 4.1.

Proof of Theorem 4.2. We consider the long exact sequence of the pair $(Z, A)$ as the spectral sequence for the filtration, we get:

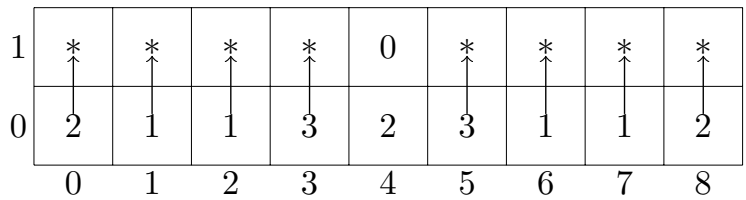

where each of the star entries is either 0 or 1 by Lemma 4.5. Lemma 4.6 implies that the first four stars are 1 and are killed by the boundary map. Inequality (2) then implies that the remaining four stars are also 1 , but are not killed by the boundary map. Thus the $E_{2}$ page looks like

\begin{tabular}{l|l|l|l|l|l|l|l|l|l|}
\hline 0 & 0 & 0 & 0 & 0 & 1 & 1 & 1 & 1 \\
\hline 1 & 0 & 0 & 2 & 2 & 3 & 1 & 1 & 2 \\
\hline 0 & 1 & 2 & 3 & 4 & 5 & 6 & 7 & 8 \\
\hline
\end{tabular}

completing the proof.

Proof of Proposition 4.1. The identity component is identified with

$$
H_{\text {oom }}\left(\pi_{1}(K l), U(2)\right) \cong X:=\left\{\left(g_{0}, g_{1}\right) \in U(2)^{2} \mid g_{0}^{2}=g_{1}^{2}, \operatorname{det}\left(g_{0}\right)=\operatorname{det}\left(g_{1}\right)\right\} .
$$

It follows that the map $\rho: X \rightarrow U(1), \rho\left(\left(g_{0}, g_{1}\right)\right)=\operatorname{det}\left(g_{0}\right)$ is a fibre bundle with fibre $Z=H o m\left(\pi_{1}(K l), S U(3)\right)$. We deduce that $\kappa: Z \times U(1) \rightarrow X$ sending $\kappa\left(\left(g_{0}, g_{1}\right), \lambda\right)=$ $\left(\lambda g_{0} . \lambda g_{1}\right)$ is a Galois cover. The deck transformation group $\mathbb{Z}_{3}=\left\{\gamma \in U(1) \mid \gamma^{3}=\mathbb{1}\right\}$ acts by $\gamma \cdot\left(\left(g_{0}, g_{1}\right), \lambda\right)=\left(\left(\gamma g_{0}, \gamma g_{1}\right), \gamma^{2} \lambda\right)$. Thus $H^{*}(X)=H^{*}(Z \times U(1))^{\mathbb{Z}_{3}}$.

To prove the proposition, we must show that $\mathbb{Z}_{3}$ acts trivially on the cohomology of $Z \times U(1)$. The action on the $U(1)$ factor is by translation, which necessarily is cohomologically trivial. To understand the effect on $H^{*}(Z)$, notice that the action preserves the subspace $A \subset Z$ from (4) Thus the long exact sequence of the pair :

$$
\ldots \rightarrow H^{*}(Z, A) \rightarrow H^{*}(Z) \rightarrow H^{*}(A) \rightarrow \ldots
$$

is acted on equivariantly by $\mathbb{Z}_{3}$. By Lemma 4.5 , the cohomology groups $H^{*}(Z, A)$ have dimension 1 or 0 in all degrees, so $\mathbb{Z}_{3}$ must act trivially on $H^{*}(Z, A)$. The action on $A$ lifts to the model space $\left(G / T \times T \times T_{2}\right)^{W}$ where it acts by translation of the $T$ factor, which is an isotopy and hence trivial cohomologically. Since $\mathbb{Z}_{3}$ acts trivially on both $H^{*}(Z, A)$ and $H^{*}(A)$, we infer from the long exact sequence that it acts trivially also on $H^{*}(Z)$, completing the proof. 


\section{References}

[1] T. Baird. The moduli space of flat SU(2)-bundles over a nonorientable surface. to appear in the Quarterly Journal of Mathematics.

[2] T.J. Baird. Cohomology of the space of commuting n-tuples in a compact Lie group. Algebraic \& Geometric Topology, 7:737-754, 2007.

[3] A. Borel. Seminar on Transformation Groups. Princeton University Press, 1960.

[4] D.S. Freed, M.J. Hopkins, and C. Teleman. Consistent orientation of moduli spaces. arXiv:0711.1909, 2007.

[5] W. Greub, S. Halperin, and R. Vanstone. Connections, curvature and cohomology, III. Academic Press, 73:76, 1972.

[6] N.K. Ho and C.C.M. Liu. Antiperfect Morse stratification. arXiv:0808.3974, 2008.

[7] N.K. Ho and C.C.M. Liu. Yang-Mills connections on nonorientable surfaces. Communications in Analysis and Geometry, 16(3):617-679, 2008.

[8] N.K. Ho, C.C.M. Liu, and D. Ramras. Orientability in Yang-Mills theory over nonorientable surfaces. arXiv:0810.4882, 2008.

Mathematical Institute, University of Oxford, 24-29 St. Giles' St., Oxford UK, OX1 3LB

E-mail address: baird@maths.ox.ac.uk 